\title{
PARTS OF MEASURES AND INTEGER-VALUED TRANSFORMS
}

\author{
BY LOUIS PIGNO
}

Communicated by Richard R. Goldberg, September 20, 1976

In this paper $G$ is a compact abelian group with ordered dual $\Gamma$. By this we mean there is a nontrivial group homomorphism $\phi: \Gamma \rightarrow \mathbf{R}$ where $\mathbf{R}$ is the additive group of real numbers. Let $M(G)$ be the usual convolution algebra of finite Borel measures on $G$ and ^ the Fourier-Stieltjes transformation.

A measure $\mu \in M(G)$ is said to vanish at infinity in the direction of $\phi$ if $\left\{\gamma_{n}\right\}_{1}^{\infty} \subset \Gamma$ with $\phi\left(\gamma_{n}\right) \rightarrow \infty \Rightarrow \hat{\mu}\left(\gamma_{n}\right) \rightarrow 0$. The subspace consisting of all measures whose transforms vanish at infinity in the direction of $\phi$ will be denoted by $M_{\phi}(G)$.

Let $\delta_{0}$ be the identity measure in $M(G)$ and for any integer $N_{i}$ put $\delta_{i}=$ $N_{i} \delta_{0}$. The purpose of this note is to announce the following results which explicate a line of research begun by $\mathrm{H}$. Helson [2] and continued by various authors in [1], [3], [5], [6], and [7].

THEOREM 1. Let $\mu \in M(G)$ such that the convolution product $\Pi_{i=1}^{m}\left(\mu-\delta_{i}\right)$ $\in M_{\phi}(G)$. Then $\mu$ has a decomposition $\mu=\mu_{0}+\mu_{\perp}$ where $\mu_{0} \in M_{\phi}(G), \mu_{\perp} \in$ $M_{\phi}^{\perp}(G)$ and $\hat{\mu}_{\perp}(\Gamma) \subset\left\{N_{1}, \ldots, N_{m}\right\}$. If $\Pi_{i=1}^{m}\left(\mu-\delta_{i}\right) \in M_{0}(G)$ then $\mu$ has a decomposition $\mu=\mu_{0}+\mu_{\perp}$ where $\mu_{0} \in M_{0}(G), \mu_{\perp} \in M_{0}^{\perp}(G)$ and $\hat{\mu}_{\perp}(\Gamma) \subset$ $\left\{N_{1}, \ldots, N_{m}\right\}$. Here $M_{0}(G)$ is the ideal of measures $\mu \in M(G)$ such that $\hat{\mu} \in$ $C_{0}(\Gamma)$.

The proof of Theorem 1 is obtained by analyzing $\mu_{\perp}$ in $M(\mathbf{S})$ where $\mathbf{S}$ is the structure semigroup of $M(G)$.

Assume $\phi$ is an isomorphism, $P$ the positive cone and $\mathbf{E}$ a Sidon subset of $\Gamma$. For any subset $A$ of $\Gamma$ put $\mathbf{F}(A)=\{\mu \in M(G): \hat{\mu}$ is integer-valued on $A\}$ and $\mathrm{I}(A)=\{\mu \in M(G): \hat{\mu}=0$ or 1 on $A\}$. The following theorem is a consequence of Theorem 1 and is an extension of a result announced by I. Kessler [3]; see also [4, pp. 206-211].

Theorem 2. If $\mu \in \mathbf{F}(\Gamma \backslash-P \cup \mathbf{E})$ then there is $a \nu \in \mathbf{F}(\Gamma)$ such that $\hat{\mu}=$ $\hat{\nu}$ off $-P \cup \mathbf{E}$. In particular, if $\mu \in \mathbf{I}(\Gamma \backslash-P \cup \mathbf{E})$ then $\nu \in \mathbf{I}(\Gamma)$.

Measures such that $\hat{\mu}(\gamma)=\hat{\mu}^{2}(\gamma)$ for all $\gamma \in P$ are called semi-idempotents. A subset $\Re$ of $\Gamma$ is said to be a weak Rajchman set if supp $\hat{\mu} \subset \Re \Rightarrow \hat{\mu} \in C_{0}(\Gamma)$. An easy consequence of Theorem 1 is the following result.

AMS (MOS) subject classifications (1970). Primary 43A25. 
THEOREM 3. If $\mu \in \mathbf{F}(\Gamma \backslash \Re)$ then there is a $\nu \in \mathbf{F}(\Gamma)$ such that $\hat{\mu}=\hat{\nu}$ off R. In particular, if $\mu \in \mathbf{I}(\Gamma \backslash \Re)$ then $\nu \in \mathbf{I}(\Gamma)$.

For examples of Rajchman sets, the reader is referred to [5]. Proofs of our results will appear elsewhere.

\section{REFERENCES}

1. K. deLeeuw and Y. Katznelson, The two sides of a Fourier-Stielties transform and almost idempotent measures, Israel J. Math. 8 (1970), 213-229. MR 43 \#818.

2. H. Helson, On a theorem of Szegö, Proc. Amer. Math. Soc. 6 (1955), 235-242. MR 17, 597.

3. I. Kessler, Semi-idempotent measures on abelian groups, Bull. Amer. Math. Soc. 73 (1967), 258-260. MR 34 \#6446.

4. Y. Meyer, Algebraic numbers and harmonic analysis, North-Holland, Amsterdam, 1973.

5. L. Pigno, Fourier-Stielties transforms which vanish at infinity off certain sets, Glasgow Math. J. (to appear).

6. - Integer-valued Fourier-Stielties transforms, Technical Report, no. 53, Kansas State Univ., 1976.

7. W. Rudin, Permutations of Taylor coefficients of bounded functions, Duke Math. J. 28 (1961), 537-543. MR 25 \#5166.

DEPARTMENT OF MATHEMATICS, KANSAS STATE UNIVERSITY, MANHATTAN, KANSAS 66506 